\title{
The common zoonotic protozoal diseases causing abortion
}

\author{
Raafat Mohamed Shaapan
}

Received: 20 December 2014 / Accepted: 6 February 2015/Published online: 14 March 2015

(C) Indian Society for Parasitology 2015

\begin{abstract}
Toxoplasmosis, neosporosis, sarcosporidiosis (sarcocystosis) and trypanosomiasis are the common zoonotic protozoal diseases causing abortion which caused by single-celled protozoan parasites; Toxoplasma gondii, Neospora caninum, Sarcocystis spp and Trypanosoma evansi, respectively. Toxoplasmosis is generally considered the most important disease that causing abortion of both pregnant women and different female animals throughout the world, about third of human being population had antibodies against $T$. gondii. The infection can pass via placenta, causing encephalitis, chorio-retinitis, mental retardation and loss of vision in congenitally-infected children and stillbirth or mummification of the aborted fetuses of livestock. Neosporosis is recognized as a major cause of serious abortion in varieties of wild and domestic animals around the world particularly cattle, the disease cause serious economic losses among dairy and beef cattle due to decrease in milk and meat production. While unlike toxoplasmosis, neosporosis is not recognized as a human pathogen and evidence to date shows that neosporosis is only detected by serology in the human population. Sarcosporidiosis also can cause abortion in animals particularly cattle, buffaloes and sheep with acute infection through high dose of infection with sarcocysts. On the other hand, humans have been reported as final and intermediate host for sarcosporidiosis but not represent a serious health problem. Trypanosomiasis by $T$. evansi cause dangerous infection among domestic animals in
\end{abstract}

R. M. Shaapan $(\square)$

Veterinary Research Division, Department of Zoonosis, National

Research Center, El-Tahrir Street, Dokki, Post Box: 12622,

Giza, Egypt

e-mail: rmshaapan2005@yahoo.com tropical and subtropical areas. Several cases of abortion had been recorded in cattle and buffaloes infected with $T$. evansi while, a single case of human infection was reported in India. Trichomoniasis and babesiosis abortion occurs with non-zoonotic Trichomonas and Babesia species while the zoonotic species had not been incriminated in induction of abortion in both animals and man. The current review article concluded that there is still need of wide scope for evaluation of the zoonotic impact and control of these diseases.

Keywords Zoonotic protozoa $\cdot$ Toxoplasma Neospora . Sarcocystis $\cdot$ Trypanosoma $\cdot$ Abortion

\section{Introduction}

Abortion is the delivery of an immature fetus, either live or dead, before the completion of the gestation period beyond the expected time of parturition as a result of failure of the mechanisms that control pregnancy. If pregnancy ends before organogenesis, it is called early embryonic death. A stillbirth is a fetus that has matured fully in uterus, but is born dead. Neonatal deaths refer to that occurring primarily within the first 7 days of life (Gelaye et al. 2014). Both noninfectious (nutritional, physical, toxic and chemical) and infectious (viral, bacterial, fungal and protozoal) etiologies can result in pregnancy loss. Infectious agents are perhaps the most frequently thought of cause of abortions in human and domestic animals (Pretzer 2008). While, there are many important protozoan parasites responsible for causing abortions, the zoonotic protozoal diseases responsible for abortion incidence in domesticated animals and or human are toxoplasmosis, neosporosis, sarcocystosis and trypanosomiasis. 
Among the induced-abortion zoonotic protozoan diseases, toxoplasmosis is a worldwide high public health significant disease caused by Toxoplasma gondii. Domestic cats and other felids are the only known final or definitive hosts (FH), man and most of warm-blooded animals as intermediate hosts (IH), which can become infected through ingestion of oocysts form contaminating food or water with cat feces or consumption of undercooked meat containing viable tissue cysts (Dubey 2010). The disease responsible for major economic losses in most classes of livestock through abortions, still birth and neonatal losses (Raeghi et al. 2011); In adult human the disease does not cause serious illness, but it can cause spontaneous abortion in infected pregnant women, blindness and mental retardation in congenitally infected children and devastating disease in those with depressed immunity and furthermore, fatal toxoplasmosis may be occurred in a patient with AIDS (Ferreira et al. 2009).

Neosporosis is one of the most common diseases causes of abortion in cattle, sheep, goats, water buffalo and camels caused by Neospora caninum, a protozoan parasite closely related to $T$. gondii, is recognized as a major cause of endemic and epidemic abortions particular, in cattle around the world and seriously impacts the economic performance of the dairy and beef industries (Dubey et al. 2007). The parasite transmitted transplacentaly from cow to calf, but cows (IH) may also become infected by the ingestion of oocysts shed by dogs or coyotes (FH), (Gavrilović et al. 2013). Although antibodies to $N$. caninum have been reported (Lobato et al. 2006), the parasite has not been demonstrated in human tissues.

Sarcosporidiosis (sarcocystosis), caused by varieties of Sarcocystis species which are obligate heteroxenous, cystforming protozoan parasites, a requisite two-host life cycle based on a prey-predator (intermediate-definitive) host relationship (Jehle et al. 2009). The disease involves abortion, reduced milk production, weight loss, and death of cattle, sheep, goats and pigs (Odening et al. 1996). In humans the disease does not represent a serious health problem, they acquire intestinal sarcosporidiosis as a final host from eating raw or undercooked meat containing mature sarcocysts of zoonotic Sarcocystits spp. (S. hominis and S. suihominis), and may also become dead-end (intermediate) hosts for non-human Sarcocystis spp. after the accidental ingestion of oocysts (Fayer 2004).

Trypanosomiasis is the disease caused by Trypanosoma spp. the most important zoonotic species are Trypanosoma bruci gambiense, Trypanosoma bruci rhodesiense, causing human African sleeping sickness and Trypanosoma cruzi, causing human American chagas disease. All the 3 species are responsible for considerable production losses and morbidity in animals, but not recorded as a cause of abortion in man and animals (Steverding 2008). While, widespread Trypanosoma evansi which latterly isolated from human case in India (WHO 2005) affected many animal species (camels, sheep cattle and buffaloes) causing abortion and transplacentlly transmitted to fetus in cattle and buffaloes (Desquesnes et al. 2013).

Trichomoniasis abortion in human and animals occurs through infection with non-zoonotic Trichomonas spp., $T$. vaginalis and $T$. foetus, respectively, while the zoonotic species (T. fragilis, T. homonis, T. tenax and T. gallinae) are not causing abortion and responsible only for gastrointestinal disease (Maritz et al. 2014). Similarly, babesiosis abortion occurs in animals through infection with non-zoonotic Babesia spp., B. bovis and B. equi, while the zoonotic species; $B$. divergens, $B$. canis and B. microti had not been incriminated in induction of abortion in both animals and man (Becker et al. 2009).

According to the above mentioned data; the objective of this review is to provide knowledge about the epidemiology of the common known zoonotic protozoal diseases causing abortion in animals and/or human, their developmental biology, life cycle, epizootology, pathogenicity, significance in public health and the recent trends in diagnosis and preventive control measures.

\section{Etiology}

Toxoplasma gondii, N. caninum, Sarcocystis $\mathrm{sp}$ and $T$. evansi are single-celled zoonotic protozoan causing abortion in animals and/or man. The first three protozoan parasites are members of the Phylum Apicomplexa which characterized by a very unique organelle called an apical complex, while Trypanosoma sp is member of Phylum Kinetoplastida where its major distinguishing feature is the presence of a kinetoplast (flagellated protozoa). The scientific taxonomy of these parasites is briefly presented as following:

\begin{tabular}{|c|c|c|c|c|}
\hline Domain & \multicolumn{4}{|l|}{ Eukaryota } \\
\hline Kingdom & \multicolumn{4}{|c|}{$\begin{array}{l}\text { Chromalveolata } \\
\text { (protozoa) }\end{array}$} \\
\hline Phylum & \multicolumn{3}{|l|}{ Apicomplexa } & Kinetoplasta \\
\hline Class & \multicolumn{3}{|l|}{ Conoidasida } & Kinetoplastida \\
\hline Order & \multicolumn{3}{|c|}{ Eucoccidiorida } & Trypanosomatida \\
\hline Family & \multicolumn{3}{|l|}{ Sarcocystidae } & Trypanosomatidae \\
\hline Subfamily & \multicolumn{2}{|c|}{ Toxoplasmatinae } & Sarcocystinae & \\
\hline Genus & Toxoplasma & Neospora & Sarcocystis & Trypanosoma \\
\hline Species & gondii & caninum & $\begin{array}{l}\text { cruzi, } \\
\quad \text { hominis, } \\
\quad \text { suihominis }\end{array}$ & evansi \\
\hline
\end{tabular}

Scientific taxonomy of protozoa by Cavalier-Smith (1993) 


\section{Life cycle, infectious stages and mode of transmission}

Toxoplasma gondii

Life cycle of T. gondii

The life cycle of $T$. gondii summarized and illustrated in Fig. (1). It has a typical coccidian life cycle, cats are the definitive host, and warm-blooded animals and man as IHs, the cycle firstly was described by Frenkel et al. (1970) and further details were cleared in recent review (Ajioka and Soldati 2007).

\section{T. gondii infective stages}

There are three infectious stages of $T$. gondii: (1) Tachyzoites were found free or in inside the leucocytes, banana shape pointed at one end and rounded at the other one, their sizes mean $2 \times 6 \mu$ (Fig. 2a). (2) Tissue cysts, sub-spherical in shape with thin elastic cyst wall $50-200 \mu$ in diameter and contain several hundreds crescent shaped bradyzoites (Fig. 2b). (3) Oocysts, fresh recovered from cat feces, is un-sporulated then become sporulated, $12 \times 15 \mu$. in size, each oocyst contains 2 sporocysts and each contains 4 sporozoites and residual bodies (Fig. 2c) (Shaapan and Ghazy 2007).
Mode of transmission of T. gondii

Hosts can acquire $T$. gondii infection, horizontally by ingesting of infected animals tissue containing bradyzoites or ingesting food or drink contaminated with sporulated oocysts, or vertically by trans-placental route and infection may also occur through blood transfusion or organ transplantation (Shin et al. 2009).

Neospora caninum

\section{Life cycle of $N$. caninum}

N. caninum entail a life cycle (Fig. 3), between a definitive host (dogs and some other canids) and the IH (predominantly cattle, possibly sheep or goat) (McAllister et al. 1998). Dogs excrete oocysts that become infectious within a couple of days. These may infect IH (mainly cattle) and lead to either infection with no discernible clinical symptoms, or induce abortions 2-3 weeks later, usually at about 5-7 months of gestation (Dubey et al. 2007).

\section{$N$. caninum infective stages}

There are three infectious stages of the parasite (tachyzoites, bradyzoites and sporozoites) nearly similar in shape and size to $T$. gondii stages (Fig. $4 \mathrm{a}, \mathrm{b}, \mathrm{c}$ ). Tachyzoites and
Fig. 1 Toxoplasma gondii life cycle

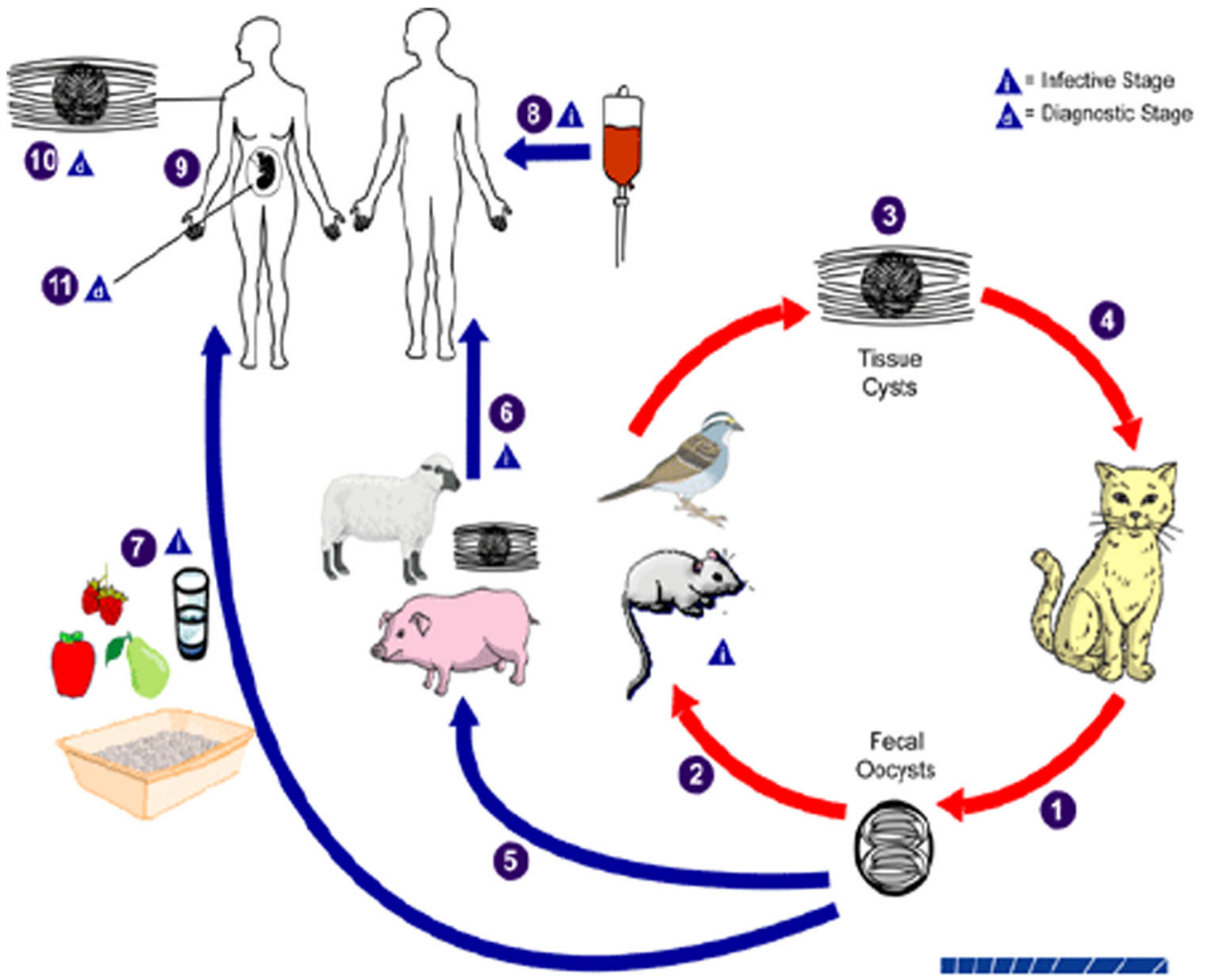



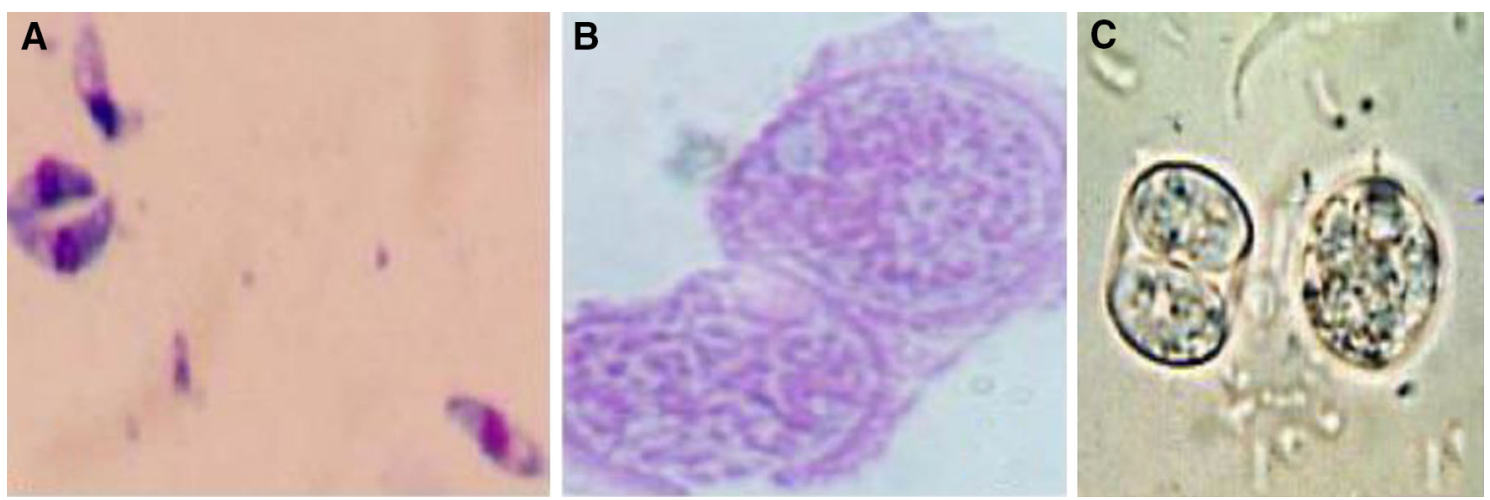

Fig. 2 T. gondii infective stages; Tachyzoites (a), tissue cysts (b) and oocysts $(\mathbf{c})(\times 400)$

Fig. 3 Neospora caninum life cycle

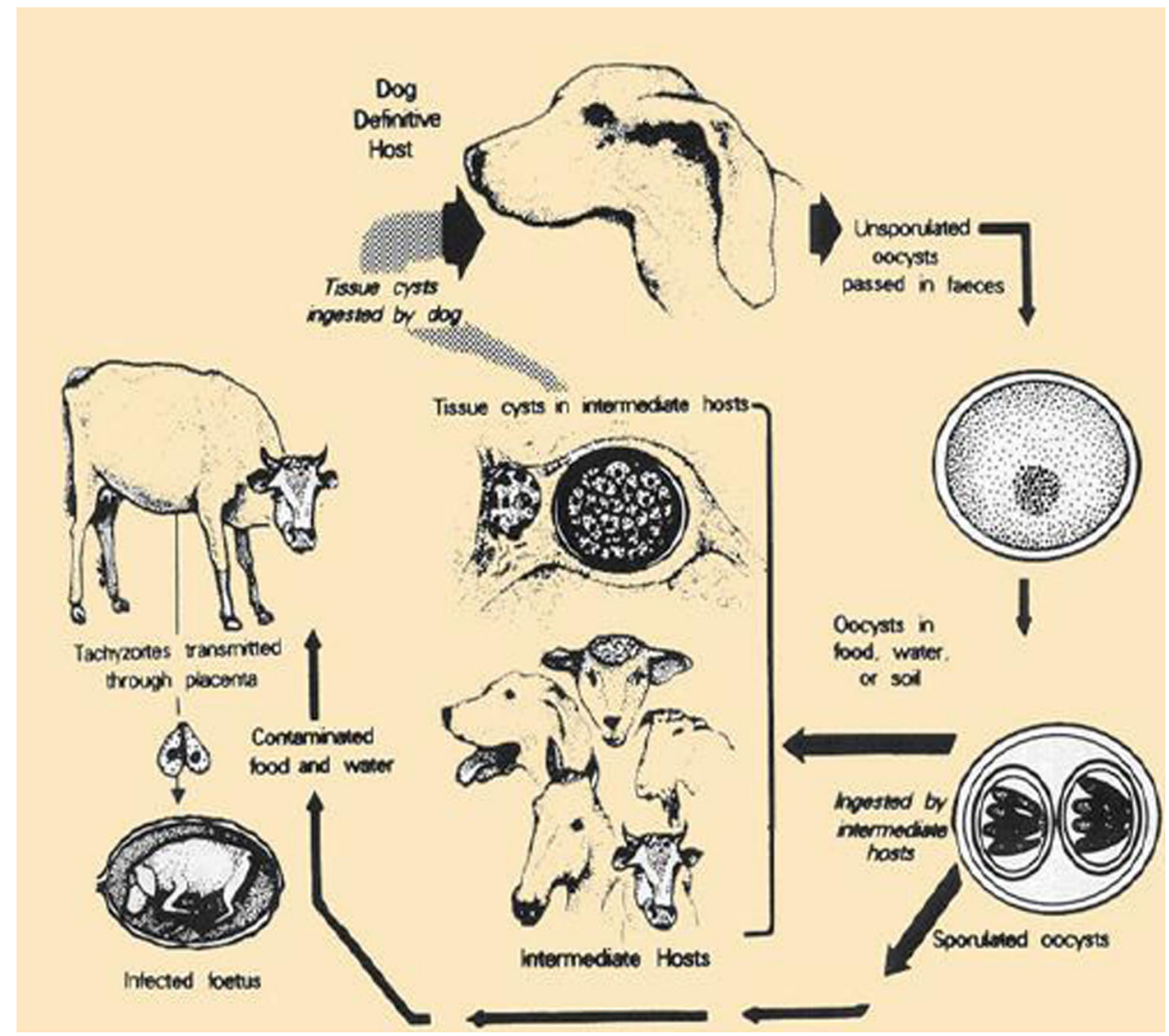

bradyzoites are asexually proliferating stages occur in tissues of infected hosts (intermediate and definitive), whereas sporozoites are present in sexually produced oocysts excreted in the definitive hosts feces (Hemphill 1999).

\section{Mode of transmission of $N$. caninum}

Dogs excrete oocysts firstly, un-sporulated and after 2-3 days become sporulated oocysts that may infect the IHs, mainly cattle and may be other mammals including man through fecal-oral route. Definitive carnivore hosts can acquire the infection by ingestion of tissues containing tissue cysts. Infected dams infect their offspring in utero mode (transplacental transmission) that appears to be highly efficient (Davison et al. 1999).

\section{Sarcocystis sp}

\section{Life cycle of Sarcocystis}

All species of Sarcocystis have obligate two host life cycles (Fig. 5); the $\mathrm{IH}$ is infected when it ingests oocysts or 

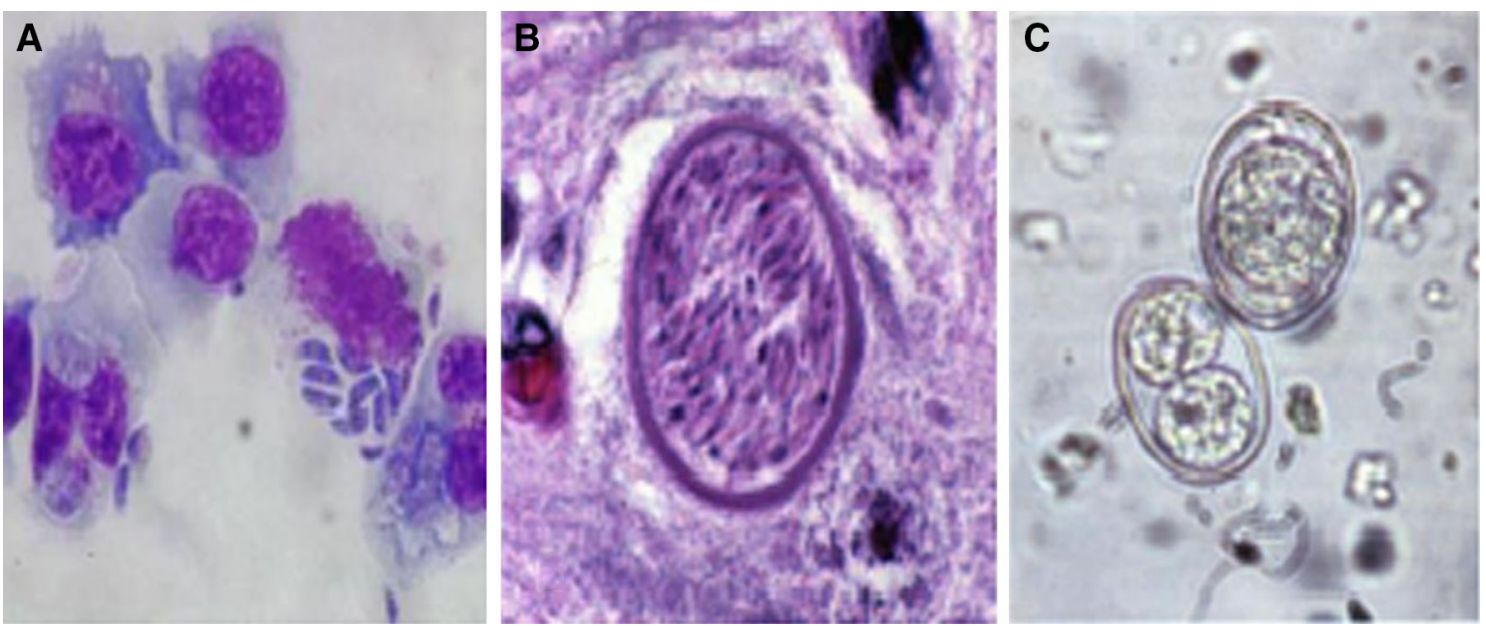

Fig. 4 Neospora caninum infective stages; Tachyzoites $(\mathbf{a})$, tissue cysts $(\mathbf{b})$ and un-sporulated and sporulated oocysts $(\mathbf{c})(\times 400)$

Fig. 5 Srcocystis sp. life cycle

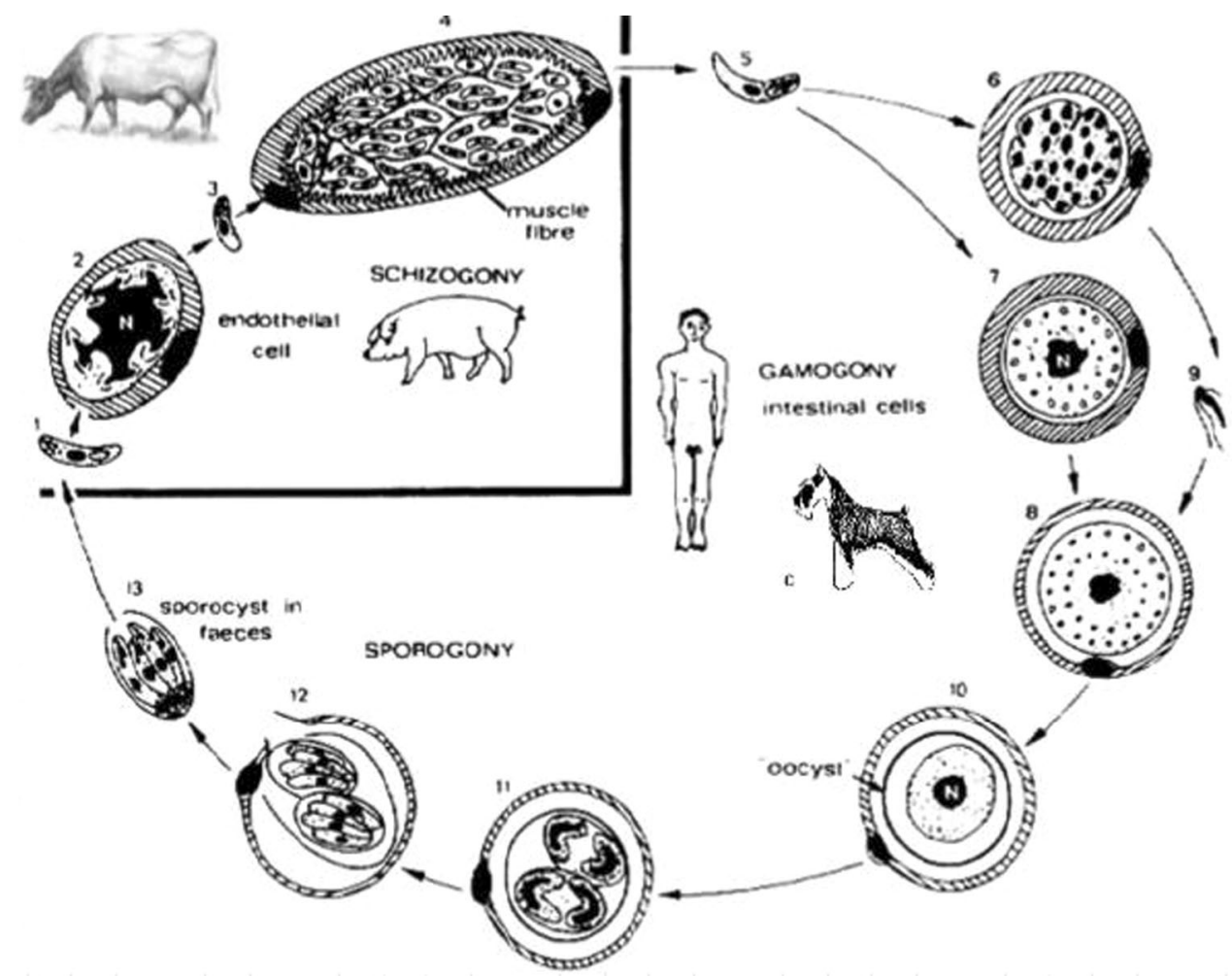

sporocysts that have been passed in the definitive host's feces. Ocysts and sporocysts excyst in intestine release the sporozoites which penetrate it distributed to tissues then the parasite reproduces asexually resulting in sarcocysts filled with bradyzoites and called zoitocysts or sarcocysts. The definitive host is infected when ingests tissues (meat or prey) containing sarcocysts, the bradyzoites liberate, infect the intestinal epithelium, reproduce sexually, forming weak walled oocysts ruptured and give sporocysts that are passed in their feces (Jehle et al. 2009).
Sarcocystis infective stages

Oocysts of Sarcocystis in FH feces measure 15-20 $\mu \mathrm{m}$, sporulated in the intestinal epithelium and contain 2 sporocysts, each contains 4 sporozoites and a refractile residual body. Due to the fragile nature of oocysts, both sporulated oocysts and individual sporocysts may be shed in feces (Fig. 6a). In muscles of IH mature sarcocysts (tissue cyst) of each species vary in size from microscopic to macroscopic, with structurally distinct sarcocysts walls 
Fig. 6 Sarcocystis sp. Infective stages; oocyst and sporocysts (a), macroscopic sarcocysts in muscles (b) and microscopic sarcocysts contain bradyzoites (c) $(\times 400)$
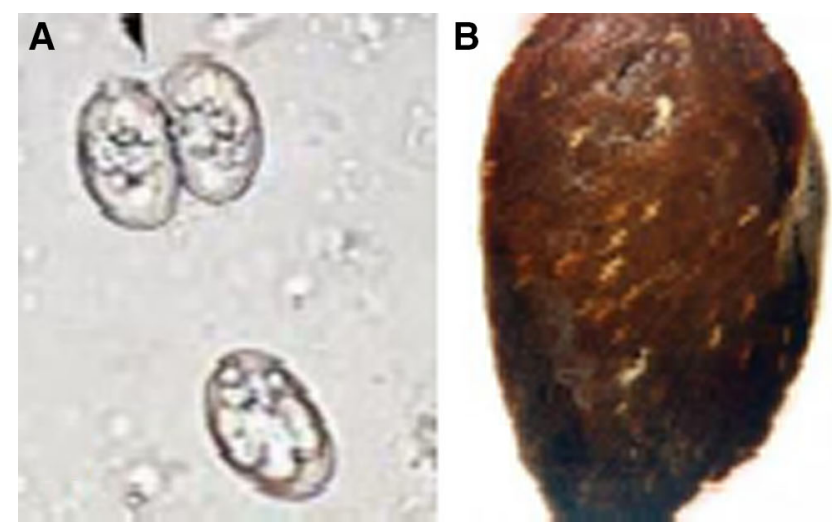

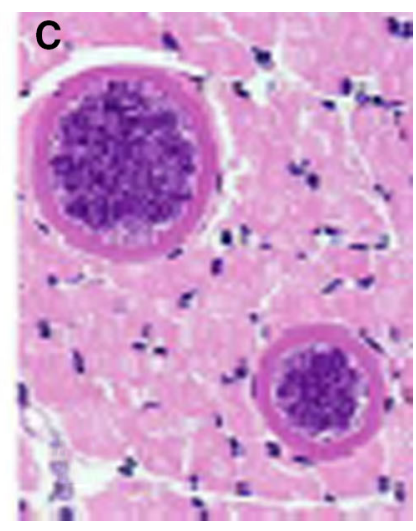

that vary in thickness and organization of villar protrusions, but all contain numerous bradyzoites (Fig. 6b, c) (Dubey et al. 1989).

\section{Mode of transmission of Sarcocystis}

The final host (predator) is infected when ingests raw tissues or meat contain the sarcocysts filled with bradyzoites, while the IH (prey) is infected with ingestion of contaminated food or water with the oocysts or sporocysts passed in FH feces (Ortega-Mora et al. 2007).

\section{Trypanosoma evansi}

\section{Life cycle and morphology of T. evansi}

Trypanosoma evansi exhibits direct life cycle with no IH, it transmitted from animal to animal mechanically by transmitting vectors in which it is not capable of cyclical development. T. evansi infected hosts comprises domesticated animals: equines, cattle, buffalo, camels, pigs, sheep, goats, dogs, cats and also human infection reported in India (Truc et al. 2007).

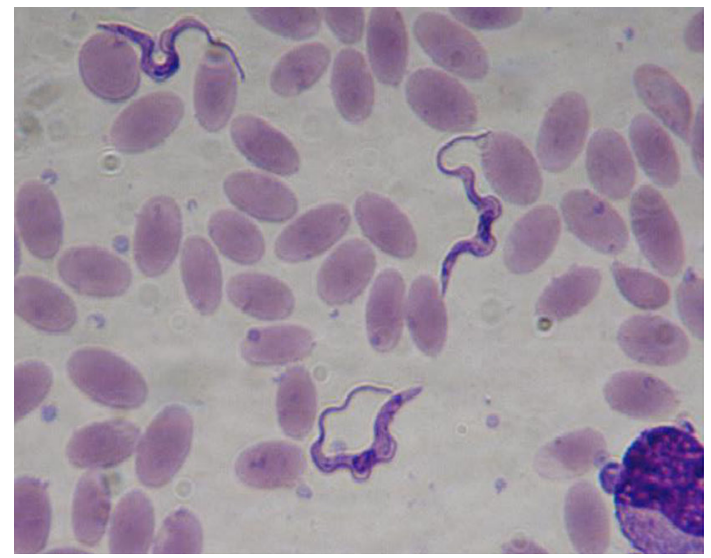

Fig. 7 T. evansi trypomastigote in Giemsa-stained blood smear
The intracellular life cycle stages are different forms of the trypomastigote found in blood and tissues of infected host. While other stages (amastigote, promastigote and meta-cyclic) not recognized due to loss of cyclical development by flies which only act as mechanical vector. The trypomastigote forms measure, $14-33 \mathrm{~mm}$ in length with a width of $1.5-2.2 \mathrm{~mm}$ (Fig. 7). They possess a free flagellum and loss of sub-terminal kinetoplast (dys-kinetoplastic forms), due to absence of kinetoplast DNA (kDNA) (Artama et al. 1992).

\section{Mode of transmission of T. evansi}

Trypanosoma evansi is transmitted in several ways; mechanically via many biting or sucking insects (Tabanus, Haematobia, Hippobosca and Stomoxys) (Fig. 8a, b, c, d), vampire bats, vertical (transplacental) transmission from dam to calf or foal, and per-oral when carnivores eat infected prey, rarely through the use of non-sterile surgical instruments during mass treatment and vaccination. Transmission in milk and during coitus has been also documented (Desquesnes et al. 2013).

\section{Identification and diagnosis}

Toxoplasmosis

\section{Clinical features (signs)}

The clinical signs of toxoplasmosis in man or animals are not sufficient for a definite diagnosis. Infected human showed flulike including swollen lymph nodes and muscle aches. However, infection just before or during pregnancy (congenital toxoplasmosis) lead to abortion or severe manifestation in fetus in form of mental retardation, microcephalous or hydrocephalus. Ocular toxoplasmosis leads to chorio-retinitis and immunocompromised (HIV, AIDS or organ-transplant recipients) patients undergo severe 
Fig. 8 Some of the potential vectors of Trypanosoma evansi; Tabanus (a), Haematobia (b), Hippobosca (c) and Stomoxys (d)

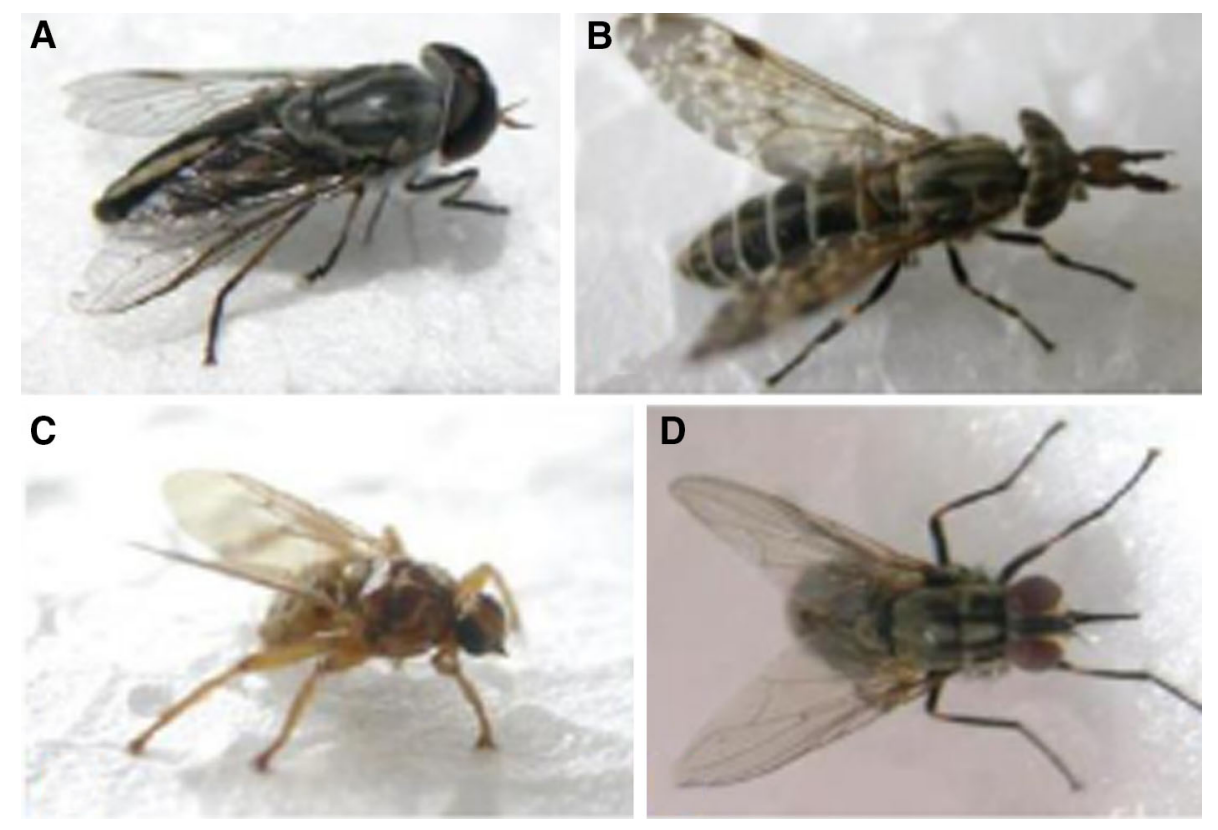

A symptoms such as damage to the eye, necrotic intracerebral mass lesions end by fatal encephalitis (Shin et al. 2009). The most serious symptoms in animal occur in pregnant sheep or goat in form of abortions, stillborn or mummified fetus with focal white spots of necrosis in placental cotyledons (Dubey 2010).

\section{Biologic diagnosis (microscopy findings)}

Direct visualization of the parasite infective stages; tachyzoites, tissue cysts with bradyzoites and oocysts in biopsy (ant-mortem) or autopsy (post-mortem) examined material specimens under the microscope, these techniques are used less frequently because of the difficulty of obtaining these specimens and need specialized laboratories (Smith 1995). T. gondii infective stages can be isolated from blood, body fluids or even some tissues after digestion; through intra peritoneal inoculation of mice and tachyzoites detected in the peritoneal fluid 6-8 days post inoculation (PI) or tissue cysts detected 6-8 weeks PI in brain print. Tachyzoites also may be isolated by tissue culture inoculation. $T$. gondii oocysts can be detected in cat fecal smears, firstly un-sporulated and after 3-5 days become sporulated (Dubey 2009).

\section{Antibody detection (serology)}

The detection of Toxoplasma-specific antibodies is the primary diagnostic method to determine infection with $T$. gondii either as an ant-mortem or post-mortem routine method of diagnosis and results require careful interpretation with a reference laboratory results. In general, Toxoplasma-specific IgG antibody appears $2-3$ weeks after acute infection, and persists for lifelong; IgM antibody suggests early infection and presence of $\operatorname{IgA}$ antibodies associated with acute infection (Wilson et al. 2003). However, several serologic tests are used for detection of anti-toxoplasma antibodies in animal and human sera such as Sabin-Feldman dye test (DT), indirect haem agglutination test (IHA), latex agglutination test (LAT), indirect fluorescent antibody test (IFAT), enzyme linked immunosorbant assay (ELISA) and modified agglutination test (MAT), the sensitivity and specificity of these test are variable. Using the more sensitive and somewhat specific MAT and/or ELISA for the detection of $T$. gondii antibodies is more practical and significant (Shaapan et al. 2008).

\section{Immuno-affinity purification and Immuno-blot}

To increase the diagnostic potency of antigens, isolation of their immunogenic fractions will be useful, tachyzoites stage is thought to be responsible for acute infection and induce strong immune responses so partial purification of tachyzoites antigen was conducted by affinity column chromatography, and the purified fraction proved potency in detecting IgG antibody level in tested sera by ELISA (Abdel-Rahman et al. 2005).

\section{Histopathology and immunohistochemistry}

Typically, macroscopically gross lesions are apparent, so histological and subsequently immunohistochemical investigations on the submitted examined biopsy or autopsy samples especially from LN, aborted fetal tissues and placenta are useful for diagnosis of toxoplasmosis by 
detection of pathological tissue lesions or $T$. gondii infective stages. In addition the immunohistochemistry technique detect the antigen in the tissue after labeled by conjugated immune-staining Ab (Ferguson et al. 2013).

\section{Molecular diagnosis (detection of T. gondii DNA)}

Detection of $T$. gondii DNA by polymerase chain reaction (PCR) is first reported using the tachyzoites B1 gene and several subsequent PCR tests have been developed using different gene targets like SAG1, SAG2 genes (Burg et al. 1989). This technique has proven very useful in direct detection of the parasite DNA extracted from different types of fresh biological samples, such as blood, aborted fetal tissues including the placenta, amniotic fetal fluid, brain, heart, skeletal muscle and even contaminated water and foods samples where the $T$. gondii stages present in low numbers and rapid and sensitive methods are necessary to detect the infection (Buchbinder et al. 2003).

\section{Neosporosis}

\section{Clinical features (signs)}

Clinical symptoms in dogs, FH, observed mainly in young pups, include hind limb paresis, paralysis with the complete loss of muscle function for one or more muscle groups (Reichel et al. 2007). In livestock IH animals, abortions are often the only sign of infection particularly in cattle at mid-term gestation (5-6 months) and up to $95 \%$ of calves congenitally infected from seropositive cows clinically healthy; rarely, have nervous signs varying from mild ataxia to tetra paralysis with increased mortality, hydrocephalus, exophthalmia and difficulty in growth (Toolan 2003).

\section{Biologic diagnosis (microscopy findings)}

Identification of $N$. caninum infective stages such as tachyzoites, bradyzoites from biopsy or autopsy of infected $\mathrm{IH}$, livestock, and oocysts from $\mathrm{FH}$, dog fees can be made by direct examination under microscope or indirectly after inoculation into mice or inoculation into specific alpaca cell line cultures (Franceschi et al. 2014).

\section{Antibody detection (serology)}

Several serological tests are used to diagnose $N$. caninum infection include IFAT, ELISA and specific neospora agglutination test (NAT). Sera and body fluids of pregnant animals or even of aborted fetuses were tested. Tachyzoites, either crude or purified and recombinant proteins are used as antigen, whereas its quality influences the specificity and sensitivity of the reaction and the falsepositive results due to antigenic cross-reactivity (OrtegaMora et al. 2006).

\section{Histopathology and immunohistochemistry}

One of the most widely used techniques for detection of neosporosis induced-abortion is the fetal histopathology combined or not with immunohistochemical examinations of biopsy or post-mortem samples from placenta, fetus brain and some other organs such as heart and liver in an attempt to detect characteristic focal necrosis lesions, positive infected tissues and may the parasite infective stages (Wouda et al, 1997).

\section{Molecular diagnosis (detection of $N$. caninum DNA)}

Serological diagnosis is complicated by the false-positive results due to antigenic cross-reactivity, therefore, different PCR techniques have been used as a more sensitive and confirmatory technique to verify the diagnosis by detection of parasite-DNA in target tissues particularly of aborted fetuses (Santos et al. 2011).

\section{Sarcocystosis}

\section{Clinical features (signs)}

Both infected FH, carnivore animal or human revealed signs that appeared 3-6 $\mathrm{h}$ after eaten raw meat containing sarcocysts include, nausea, abdominal pain, diarrhea, and eosinophilia; however symptoms in IH livestock animals varies, sever acute sarcocystosis as weakness, fever, abortion in pregnant animals, and sometimes death due to high dose of sarcocysts infection, while mild chronic sarcocystosis is caused by low dose (O'Donoghue and Rommel 1992). Accidentally infected human by ingestion of sporocysts act as $\mathrm{IH}$, most are without symptoms or showed inflammatory response to the intramuscular cysts as painful swelling, myositis, vasculitis and fever (Fayer 2004).

\section{Biologic diagnosis (microscopy findings)}

Characteristic weak wall oocysts or individual sporocysts detected in feces of FH which is easily identified microscopically after fecal floatation method. Intramuscular sarcocystosis would be suspected based on presence of myalgia, subcutaneous nodules, eosinophilia, and elevated creatinine kinase levels, sarcocysts isolated from infected tissues by biopsy (ant-mortem) or animal carcass (postmortem) which may macroscopical visible by naked eye or microscopical sarcocysts seen under microscope after 
pepsin digestion of infested muscle or tissue (Dubey et al. 1989).

\section{Antibody detection (serology)}

Sarcocystis-specific antibodies can be detected using several serological tests mainly ELISA and IFAT which show a high sensitivity for the late phase of infection. These tests are not species-specific because traditional antigen preparations derived from merozoites of any heterologous Sarcocystis species (Tenter 1995).

\section{Histopathology and immunohistochemistry}

Sarcocystis sarcocysts in muscle biopsy specimens can be identified by microscopic examination of histologic sections stained with hematoxylin and eosin (H\&E) and other stains such as the periodic acid-Schiff (PAS) which give positive reaction with cyst wall and confirmatory immunohistochemistry stain can be performed. Sarcocysts had infrequently accompanied with necrosis, perivascular and interstitial inflammation, vasculitis, and eosinophilic myositis (Arness et al. 1999).

\section{Molecular diagnosis (detection of Sarcocystis DNA)}

Much more sensitive and accurate PCR amplification of the18S rRNA used for sarcocystosis infection diagnosis particularly in aborted fetal tissues and RAPD-PCR has been used for the differentiation of Sarcocystis species (Guclu et al. 2004).

\section{$\underline{\text { Trypanosomiasis }}$}

\section{Clinical features (signs)}

Animal trypanosomiasis caused by $T$. evansi showed considerable differences in the severity of clinical signs, depending on the virulence of the strain and the susceptibility of the host. Most of infected animals revealed anemia, fever, edema, enlarged lymph nodes, icterus, emaciation and death. Abortion in cattle and buffalo was detected in several cases (Gutierrez et al. 2005). The only reported human cases of $T$. evansi infection revealed parasitaemia associated with febrile episodes for several months (Truc et al. 2007).

\section{Biologic diagnosis (microscopy findings)}

Microscopic examination at by help of $100 \times$ oil immersion lens is used for the detection of trypomastiogote forms of $T$. evansi in wet films, Giemsa stained blood smears, or in buffy coat of capillary tubes after micro-haematocrit centrifugation test. These methods are specific but less sensitive, principally in detecting parasites during low parasitemia (Monzón et al. 1995).

\section{Antibody detection (serology)}

Trypanosoma evansi is detected by several serological tests such as IFAT, ELISA and the specific card agglutination test (CATT) which gives positive reaction visible to the naked eye as blue granular agglutinations. These serological tests used for $T$. evansi mass screening (Wernery et al. 2001).

\section{Molecular diagnosis (detection of Trypanosoma DNA)}

PCR test for $T$. evansi displayed at low parasitemia especially by MCHT test negative. It was performed with primer sets (TBR1 and TBR2) specific for Trypanozoon satellite DNA regions, positive results showed a single band (164 bp) only for T. evansi (Masiga et al. 1992).

\section{Differential diagnosis}

Although, abortion due to toxoplasmosis, neosporosis, sarcocystosis and trypanosomiasis in animals may be clinically similar; $T$. gondii abortion the only of them can occur in human and is frequently in sheep and goats, $N$. caninum mainly associated with cattle abortion and uncommon in sheep and goat. The abortions due to Sarcocystis sp occur only in severe infection in livestock animals and trypanosomiasis abortions also occur in severe infestation of cattle and buffaloes (Ortega-Mora et al. 2007).

The isolated infective stages of Sarcocystis sp (sarcocysts and sporocysts) and $T$. evansi (trypomastiogote forms) are characteristics and easily identified. While $T$. gondii and N. caninum infective stages (tachyzoites, tissue cysts and oocysts) also individually characteristics but are nearly similar and can be differentiated from each other antigenically and sometimes structurally (Dubey 2010).

Tachyzoites of both $N$. caninum and $T$. gondii are identical under the light microscope but can be distinguished using transmission electron microscope based on the appearance of their rhoptries, where tachyzoites of $T$. gondii are electron-dense but of $N$. caninum are honeycombed. Well-developed tissue cysts of $N$. caninum can be distinguish from $T$. gondii through the light microscopy, $N$. caninum tissue cysts occur in neural tissues, and of thick cyst wall (up to $4 \mu \mathrm{m}$ ), while tissue cysts of $T$. gondii found in many organs and tissues and of thin cyst wall $(<1 \mu \mathrm{m})$ (Montoya and Liesenfeld 2004).

The oocysts of T. gondii, N. caninum and Sarcocystis are structurally similar; composed of 2 sporocysts each contains 4 sporozoites. Cats only $\mathrm{FH}$ give $T$. gondii oocysts in 
their feces, while dogs shed oocysts of $N$. caninum, zoonotic Sarcocystis and recently mechanically shed $T$. gondii oocysts. The oocysts of Sarcocystis of weak cyst wall ruptured in in intestine and the sporocysts are the only stage found in feces. Sporulated oocysts of N. caninum in water are smaller with length/width ratio $\leq 1.06$ than that of T. gondii $>1.5$ (Schares et al. 2005).

None of the serologic tests used to detect $N$. caninum antibodies have been validated based on recovery of the viable parasite in any host. Therefore, the cutoff values used for serologic diagnosis of $N$. caninum are presumptive. Because $N$. caninum is structurally and molecularly related to $T$. gondii, these parasites are antigenically different and serologic cross-reactivity, if present, is considered minor and therefore, specific confirmatory immunohistochemistry and PCR techniques are of significant need (Unzaga et al. 2014).

However, serological tests help in differential diagnosis; serological diagnosis is complicated by the potential for false-positive results due to antigenic cross-reactivity. $N$. caninum and $T$. gondii have many shared antigens especially by ELISA, uses of specific tests as IFAT, MAT and DT showed little or no cross reactivity and also, the use of specific purified fractions from antigens will improve diagnosis using ELISA (Hassanain et al. 2013).

\section{Prevention and control}

\section{Toxoplasmosis}

\section{Serologic screening during pregnancy}

Serologic screening in women during pregnancy is important way to reduce the infection, while seropositive women must be treated to reduce congenital transmission but not clinical disease in infants (Barbosa et al. 2009).

\section{Hygiene measures}

To prevent infection of human beings by $T$. gondii, people should wash their hands thoroughly with soap and water after handling meat, also all cutting boards, knives, and other utensils that were in contact with uncooked meat should be washed (Dubey and Jones 2008). Meat should be well cooked before consumption by human or other animals, and tasting or seasoning while meat cooking should be avoided, heating at $60^{\circ} \mathrm{C}$ or more for $10 \mathrm{~min}$, freezing at either $-10{ }^{\circ} \mathrm{C}$ for 3 days or $-20^{\circ} \mathrm{C}$ for 2 days, or irradiation at doses of $75 \mathrm{rad}$ was sufficient to kill tissue cysts, meanwhile, neither cooking in a microwave nor chilling at $8{ }^{\circ} \mathrm{C}$ was sufficient to kill tissue cysts (Elnawawi et al, 2008). Pregnant women and animals should avoid contact with cat feces, and cat litter should be disposed of every day by persons other than pregnant women. Gloves must be wearing while gardening and vegetables should be washed thoroughly. Dead animals, aborted fetuses should be handled by caution, and buried or incinerated to prevent scavenging by pigs and cats (Pappas et al. 2009).

\section{Animal management practices}

Keeping cats out of the animal barns and pet cats should be fed only dry, canned, or cooked food and efforts should be made to keep it indoors to prevent hunting, to reduce feed contamination by $T$. gondii oocysts (Dubey et al. 2005).

\section{Vaccination}

Objectives for the use of vaccines against toxoplasmosis include reducing fetal damage; decrease the formation of $T$. gondii cysts in food animals and preventing oocysts shedding by cats. Achieving all these objectives in single vaccine currently is not feasible (Dubey and Jones 2008). In Europe and New Zealand, commercially available vaccine (Toxovax ${ }^{\circledR}$ ) of a live cyst-less strain (S-48) of $T$. gondii, used for sheep reduces neonatal mortality in lambs (Buxton and Innes 1995). Vaccination of cats with a live bradyzoites vaccine from a mutant strain (T-263) has been developed, to reduce oocysts shedding (Frenkel et al. 1991). Although several strategies have been suggested for making a vaccine, to date, there is no vaccine suitable for human use. DNA vaccination, decrease the cerebral cyst loads in infected mice (Rosenberg et al. 2009).

\section{Neosporosis}

Since, at present, neosporosis in human can be only serologically detected and so no special measures are recommended for human neosporosis. However, control programs for livestock neosporosis are being developed in different countries to control abortion. Comparing the expenses of control measures with the benefit of reduced economic losses due to $N$. caninum abortion must be evaluated (Reichel and Ellis 2006). The general strategies (programs) for neosporosis control in animals was reported by Dubey et al. (2007), based on:

\section{Farm biosecurity}

Farm biosecurity are the all activities undertaken to avoid the introduction of the disease include, (i) quarantine and testing of purchased cattle, (ii) prevention of dogs entrance to farms and eat aborted tissues, (iii) prevention of waterborne transmission (iv), rodent (reservoir) control and 
(v) prevention of stress factors for disease recrudescence in congenitally infected cattle.

\section{Reproductive management}

For reduction of the chances of transplacental transmission, (i) Embryo transfer from infected dam to non sero-negative one to avoid trans-placental transmission (ii) Artificial insemination of seropositive dams.

\section{Testing and culling}

Neospora caninum-infected cows are culled from the herd or not re-bred; this approach is very expensive and not economic in large infected herd.

\section{Chemotherapy}

Infected calves can be treated with toltrazuril which may show some efficacy in preventing vertical transmission in small-scale trials but is not currently recommended as a treatment in large scale.

\section{Vaccination}

An inactivated vaccine (irradiated tachyzoites) of $N$. caninum has been available commercially (Neoguard ${ }^{\circledR}$ ), but appears only partially $(50 \%)$ successful in preventing abortions in cattle. However, live vaccination, of attenuated $N$. caninum strain (NC-Nowra ${ }^{\circledR}$ ) demonstrate higher efficacy but may suffer as a commercial product from limited shelf-life and high production cost. Most studies published to date have focused on the evaluation of subunit recombinant vaccines from tachyzoites proteins for their vaccine potential despite the fact that it is still not clear whether this tachyzoites contains protective antigens (Weston et al. 2012).

\section{Sarcocystosis}

The general control strategy for sarcosporidiosis in man and animals was reported by Fayer (2004), based on:

\section{Prevention of intestinal sarcosporidiosis}

Intestinal sarcosporidiosis in predator FH (carnivores and human) can be prevented by thoroughly cooking or freezing meat to kill bradyzoites in the sarcocysts, where, pork and beef could be made safe for consumption by cooking at $70{ }^{\circ} \mathrm{C}$ for $15 \mathrm{~min}$ or by freezing at $-4{ }^{\circ} \mathrm{C}$ for 2 days or $20{ }^{\circ} \mathrm{C}$ for 1 day. Also, dogs and other carnivores should not be allowed to eat raw meat, offal, or dead animals.

\section{Prevention of muscular sarcocystosis}

Muscular sarcocystosis in IH food animals and accidentally human can be controlled by avoid them from ingestion of sporocysts or oocysts from carnivores FH feces contaminating their food. Dogs and cats should not be allowed in buildings used to store feed or house animals. Where drinking water is suspected to contaminate with carnivore feces, boiling is the best method to ensure disinfection and where contaminated foods are suspected, they should be thoroughly washed or cooked before being eaten.

\section{Prophylaxis and vaccination}

Prophylactic administrations of amprolium or salinomycin protect experimentally infected sheep, while therapeutic treatment of cattle and sheep has been in-effective and also, vaccines are not available.

\section{Trypanosomiasis}

Due to absence of trypanosomiasis vaccine, the control described by Desquesnes et al. (2013), they divided the control strategy into 3 sections.

\section{Pathogen control (use of trypanocides)}

The trypanocides to kill $T$. evansi may be either curative drug, kill the parasites but not always eliminate all of them or curative/preventive drugs, used as a chemoprophylaxis to prevent the new parasites infection.

\section{Vector control (preventive management)}

The control of $T$. evansi vector populations can be attempted by using traps and/or impregnated screens or using insecticides in form of vapors on livestock.

\section{Avoid the introduction of infected animals}

Equines and/or camels or any mammal from an infected to a non-infected country stay for 4-week quarantine. To maintain the status of non-infection, animals must be negative to $T$. evansi infection by testing twice at a 3-month interval.

\section{Conclusion and recommendations}

\section{Conclusion}

From the aforementioned data we can conclude that: 
- Toxoplasmosis, neosporosis, sarcosporidiosis and trypanosomiasis are the common known zoonotic protozoal diseases causing abortion. Toxoplasmic abortion is the only of them can occur in both human and animals (frequently in sheep and goats) and the other three protozoal diseases cause abortion only in animals, neosporosis mainly associated with cattle abortion, while sarcocystosis and trypanosomiasis abortion occur only in severe cases of infection (high infestation dose) in livestock animals.

- Typically, T. gondii causes abortion at late gestation period, placental cotyledons show macroscopically visible coagulative necrosis inform of white spots of large necrotic foci. Clinical signs of $N$. caninum abortion appear to be similar to those of toxoplasmosis, the abortion (mainly in cattle) occur at mid-term (5-6 month) to late gestation, the aborted placenta revealed focal necrosis and characteristic non-suppurative inflammatory lesions in the heart and mineralization in the brain of aborted fetus. Sarcocystis (in most livestock) and T. evansi (cattle and buffaloes) cause of abortion only in severe infection.

- Due the clinical sings resemblance of abortion caused by protozoal infections, thus the isolation of parasite infective stages is of necessary, Sarcocystis sp (sarcocysts and sporocysts) and T. evansi (trypomastiogote) stages are easily identified, while $T$. gondii and $N$. caninum infective stages (tachyzoites, tissue cysts and oocysts) are structurally nearly similar, so serological identification and confirmatory molecular technique (PCR) is of significant need.

- In my opinion, there is still need of wide scope for evaluation of the zoonotic impact and control of such diseases, further research is required to given the areas of risk from acute Sarcocystis and T. evansi infection in developing countries, and moreover, further investigation into the importance of zoonotic Trichomonads and Basesia for human health and its relation to abortion.

\section{Recommendations}

In a view of the epidemiology and public health significance of induced abortion protozoa; T. gondii, N. caninum, Sarcocystis and T. evansi the following points are recommended to reduce the transmission of such parasites:

(1) Improve the educational status of the people and teaching them to be aware from the abortion-inducing zoonotic protozoa with reference to its life cycle.

(2) Thoroughly wash of hands, cutting boards, knives and materials in contact with the uncooked meat with soap and water as well as fresh vegetables should be washed.

(3) Care should be taken when in contact with aborting animals or handling the aborted material and in addition, suspected affected animal should be isolated and the aborted material safely disposed.

(4) Dogs, cats must be kept away from animal farms to reduce contamination with oocysts and also, eradication of stray dogs, cats, reservoir rodents, cockroaches and flies.

(5) For control toxoplasmosis abortion; pregnant women should not eat or taste undercooked meat and also avoid handling cat litter, serological investigation advised as a routine for pregnant, indoor cat fed only dry canned or boiled food and vaccination of cats by T-263 strain to prevent oocyst shedding and food animals by $\mathrm{S}-48$ strain to reduce abortion and human risk by decrease cyst formation.

(6) Neosporosis abortion controlled through, serological testing then culling of infected cattle and quarantine followed replacement of infected purchased cattle, preventing dogs and other potential definitive hosts from enter animal houses transfer of embryos from infected cows to uninfected recipients.

(7) Sarcocystosis avoidance are: dogs and other carnivores should not eat raw meat, offal, or dead animals. Prevention of intestinal sarcosporidiosis in FH, carnivores and human by thoroughly cooking of meat at $70{ }^{\circ} \mathrm{C}$ for $15 \mathrm{~min}$ or by freezing at $-20^{\circ} \mathrm{C}$ for 1 day to kill the sarcocysts. In Egypt, a majority of food animals are infected with Sarcocystis and meat from these animals could represent a source of human infections, so cooking of the meat is recommended way to will kill the sarcocysts.

(8) Abortion by $T$. evansi prevented through, use of curative or curative/preventive trypanocidal drugs to kill and prevent the new parasites infection. Control $T$. evansi vector populations by using traps and/or impregnated screens or using insecticides in form of vapors on livestock. Quarantine and testing for suspected animals from an infected to a non-infected country to avoid spread of infection.

(9) Trichomoniasis and babesiosis abortions in human and animals occur through infection with nonzoonotic strains, on the other hand, the zoonotic strains are not incriminated in induction of abortion, therefore, these observations prompt further investigation into their importance for human health and its relation to abortion. 


\section{References}

Abdel-Rahman EH, Shaapan RM, Ghazy AA (2005) Prevalence of antibodies to Toxoplasma gondii in farm horses in Egypt. J Egypt Vet Med Assoc 65:99-112

Ajioka JW, Soldati D (2007) Toxoplasma molecular and cellular biology. Horizon Bioscience, Norfolk, pp xii-xviii

Arness MK, Brown JD, Dubey JP, Neafie RC, Granstrom DE (1999) An outbreak of acute eosinophilic myositis due to human Sarcocystis parasitism. Am J Trop Med Hyg 1:548-553

Artama WT, Agey MW, Donelson JE (1992) DNA comparisons of Trypanosoma evansi (Indonesia) and Trypanosoma brucei spp. Parasitology 104(01):67-74

Barbosa IR, de Carvalho CM, Holandab X, de Andrade-Neto VF (2009) Toxoplasmosis screening and risk factors amongst pregnant females in Natal, northeastern Brazil. Trans R Soc Trop Med Hyg 103:377-382

Becker CA, Bouju-Albert A, Jouglin M, Chauvin A, Malandrin L (2009) Natural transmission of zoonotic Babesia spp. by Ixodes ricinus ticks. Emerg Infect Dis 15(2):320-322

Buchbinder S, Blatz R, Rodloff AC (2003) Comparison of real-time PCR detection methods for B1and P30 genes of Toxoplasma gondii. Diagn Microbiol Infect Dis 45:269-271

Burg JL, Grover CM, Pouletty P, Boothroyd JC (1989) Direct and sensitive detection of a pathogenic. protozoan, Toxoplasma gondii, by polymerase chain reaction. J Clin Microbiol 27:1787-1792

Buxton D, Innes EA (1995) A commercial vaccine for ovine toxoplasmosis. Parasitology 110:511-516

Cavalier-Smith D (1993) Kingdom protozoa and its 18 phyla. Microbiol Rev 57:953-994

Davison HC, Otter A, Trees AJ (1999) Estimation of vertical and horizontal transmission parameters of Neospora caninum infections in dairy cattle. Int J Parasitol 29:1683-1689

Desquesnes M, Dargantes A, Lai DH, Lun ZR, Holzmuller P, Jittapalapong S (2013) Trypanosoma evansi and surra: a review and perspectives on transmission, epidemiology and control, impact and zoonotic aspects. Bio Med Res Int 2013:1-20

Dubey JP (2009) History of the discovery of the life cycle of Toxoplasma gondii. Int J Parasitol 39:877-882

Dubey JP (2010) Toxoplasmosis of animals and humans, 2nd edn. CRC Press, Boca Raton. ISBN 978-1-4200-9236-3

Dubey JP, Jones JL (2008) Toxoplasma gondii infection in humans and animals in the United States. Int $\mathbf{J}$ Parasitol 38(11): $1257-1278$

Dubey JP, Speer CA, Fayer R (1989) Sarcocystis of animals and man, 3rd edn. CRC Press Inc, Boca Raton, pp 1-215

Dubey JP, Hill DE, Jones JL (2005) Prevalence of viable Toxoplasma gondii in beef, chicken and pork from retail meat stores in the United States: risk assessment to consumers. J Parasitol 91:1082-1093

Dubey JP, Schares G, Ortega-Mora LM (2007) Epidemiology and control of neosporosis and Neospora caninum. Clin Microbiol Rev 20:323-367

Elnawawi FA, Twafik MA, Shaapan RM (2008) Methods for inactivation of Toxoplasma gondii cysts in meat and tissues of experimentally infected sheep. Foodborne Pathog Dis 5:687-690

Fayer R (2004) Sarcocystis spp. in human infections. Clin Microbiol Rev 17(4):894-902

Ferguson DJ, Bowker C, Jeffery KJ, Chamberlain P, Squier W (2013) Congenital toxoplasmosis: continued parasite proliferation in the fetal brain despite maternal immunological control in other tissues. Clin Infect Dis 56(2):204-208

Ferreira MU, Hiramoto RM, Aureliano DP, da Silva-Nunes M, da Silva NS, Malafronte RS, Muniz PT (2009) A community-based survey of human toxoplasmosis in rural Amazonia: seroprevalence, seroconversion rate, and associated risk factors. Am J Trop Med Hyg 81:171-176

Franceschi V, Jacca S, Sassu EL, Stellari FF, van Santen VL, Donofrio G (2014) Generation and characterization of the first immortalized alpaca cell line suitable for diagnostic and immunization studies. PLoS One 9(8): e105643

Frenkel JK, Dubey JP, Miller NL (1970) Toxoplasma gondii in cats: fecal stages identified as coccidian oocysts. Science 167:893-896

Frenkel JK, Pfefferkorn ER, Smith DD (1991) Prospective vaccine prepared from a new mutant of Toxoplasma gondii for use in cats. Am J Vet Res 52:759-763

Gavrilović P, Živulj A, Todorović I, Jovanović M, Parunović J (2013) Investigation of importance of Neospora caninum in aetiology of abortion in dairy cows in Serbia. Revue Méd Vét 164(2):100-104

Gelaye AA, Taye KN, Mekonen T (2014) Magnitude and risk factors of abortion among regular female students in Wolaita Sodo University, Ethiopia, BMC. Women's Health 14(50):1-9

Guclu F, Aldem-R OS, Guler L (2004) Differential identification of cattle Sarcocystis Spp. by random amplified Polymorphic DNAPolymerase chain reaction (RAPD-PCR). Revue Méd Vét 155:440-444

Gutierrez C, Corbera JA, Juste MC, Doreste F, Morales I (2005) An outbreak of abortions and high neonatal mortality associated with Trypanosoma evansi infection in dromedary camels in the Canary Islands. Vet Parasitol 130(1):163-168

Hassanain MA, Abdel-Rahman EH, Toaleb NI, Shaapan RM, ElFadaly HA, Hassanain NA (2013) Development of $116 \mathrm{kDa}$ Fraction for detecting experimental Toxoplasma gondii infections in mice. Iran J Parasitol 8(3):441-448

Hemphill A (1999) The host-parasite relationship in neosporosis. Adv Parasitol 43:47-104

Jehle C, Dinkel A, Sander A, Morent M, Romig T, Luc PV, De TV, Thai VV, Mackenstedt U (2009) Diagnosis of Sarcocystis spp. in cattle (Bos taurus) and water buffalo (Bubalus bubalis) in Northern Vietnam. Vet Parasitol 166(3-4):314-320

Lobato J, Silva DAO, Mineo TWP, Amaral JDHF, Segundo GRS, Costa-Cruz JM, Ferreira MS, Borges AS, Mineo JR (2006) Detection of immunoglobulin $\mathrm{G}$ antibodies to $N$. caninum in humans: high seropositivity rates in patients who are infected by human immunodeficiency virus or have neurological disorders. Clin Vaccine Immunol 13:84-89

Maritz JM, Land KM, Carlton JM, Hirt RP (2014) What is the importance of zoonotic trichomonads for human health? Trend Parasitol 30(7):333-341

Masiga D, Smyth AJ, Hayes P, Bromidge TJ, Gibson WC (1992) Sensitive detection of trypanosomes in tsetse flies by DNA amplification. Int J Parasitol 22:909-918

McAllister MM, Dubey JP, Lindsay DS, Jolley WR, Wills RA, McGuire AM (1998) Dogs are definitive hosts of Neospora caninum. Int J Parasitol 28:1473-1478

Montoya JG, Liesenfeld O (2004) Toxoplasmosis. Lancet 363:1965-1976

Monzón CM, Hoyos CB, Jara GA (1995) Outbreaks of equine trypanosomiasis caused by Trypanosoma evansi in Formosa Province, Argentina. Rev Sci Tech 14:747-752

Odening K, Stolte M, Bockhardt I (1996) On the diagnostics of Sarcocystis in cattle: Sarcocystis of a species unusual for Bos taurus in a dwarf zebu. Vet Parasitol 66:19-24

O’Donoghue P, Rommel M (1992) Australian-German collaborative studies on the immunology of Sarcocystis infections. Angew Parasitol 33(2):102-119

Ortega-Mora LM, Fernández-García A, Gómez-Bautista M (2006) Diagnosis of bovine neosporosis: recent advances and perspectives. Acta Parasitol 51:1-14 
Ortega-Mora LM, Gottstein B, Conraths FJ, Buxton D (2007) Protozoal abortion in farm ruminants: guidelines for diagnosis and control. Oxford shire, UK: CABI, pp 123-131, 153-159, 284-287

Pappas G, Roussos N, Matthew EF (2009) Toxoplasmosis snapshots: global status of Toxoplasma gondii seroprevalence and implications for pregnancy and congenital toxoplasmosis. Int J Parasitol 39(12): 1385-1394

Pretzer SD (2008) Bacterial and protozoal causes of pregnancy loss in the bitch and queen. Theriogenology 70:320-326

Raeghi S, Akaberi A, Sedeghi S (2011) Seroprevalence of T. gondii in sheep, cattle and horses in North-West of Iran. Iran J Parasitol 6:90-94

Reichel MP, Ellis JT (2006) If control of Neospora caninum infection is technically feasible does it make economic sense? Vet. Parasitol 142:23-34

Reichel MP, Ellis JT, Dubey JP (2007) Neosporosis and hammondiosis in dogs. J Small Anim Pract 48(6):308-312

Rosenberg C, Stéphane SD, Jongert E, Gargano N, Beghetto E, Porto PD, Vorup-Jensene T, Petersena E (2009) Induction of partial protection against infection with Toxoplasma gondii genotype II by DNA vaccination with recombinant chimerical tachyzoites antigens. Vaccine 27:2489-2498

Santos DSD, Andrade MP, Varaschin MS, Guimarães AM, Hirsch C (2011) Neospora caninum in bovine fetuses of Minas Geri's, Brazil: genetic characteristics of rDNA. Rev Brasil de Parasitolo Vet 20(4):281-288

Schares G, Pantchev N, Barutzki D, Heydorn AO, Bauer C, Conraths FJ (2005) Oocysts of Neospora caninum, Hammondia heydorni, Toxoplasma gondii and Hammondia hammondi in faeces collected from dogs in Germany. Int J Parasitol 35(14): $1525-1537$

Shaapan RM, Ghazy AA (2007) Isolation of Toxoplasma gondii from horse meat in Egypt. Pak J Biol Sci 10:174-177

Shaapan RM, El-Nawawi FA, Tawfik MA (2008) Sensitivity and specificity of various serological tests for the detection of Toxoplasma gondii infection in naturally infected sheep. Vet Parasitol 153:359-362
Shin DW, Cha DY, Hua QJ, Cha GH, Lee YH (2009) Seroprevalence of Toxoplasma gondii infection and characteristics of seropositive patients in general hospitals in Daejeon, Korea. Korean J Parasitol 47:125-130

Smith JE (1995) A ubiquitous intracellular parasite: the cellular biology of Toxoplasma gondii. Int J Parasitol 25:1301-1309

Steverding D (2008) The history of African trypanosomiasis. Parasit Vectors $1(3): 1-8$

Tenter AM (1995) Current research on Sarcocystis species of domestic animals. Int J Parasitol 25:1311-1330

Toolan DP (2003) Neospora caninum abortion in cattle- a clinical perspective. Irish Vet J 56:404-410

Truc P, Gibson W, Herder S (2007) Genetic characterization of $T$. evansi isolated from a patient in India. Infect Genet Evol 7(2):305-307

Unzaga JM, Moré G, Bacigalupe D, Rambeaud M, Pardini L, Dellarupe A, De-Felice L, Gos ML, Venturini MC (2014) Toxoplasma gondii and Neospora caninum infections in goat abortions from Argentina. Parasitol Int 63(6):865-867

Wernery U, Zachariah R, Mumford JA, Luckins T (2001) Preliminary evaluation of diagnostic tests using horses experimentally infected with Trypanosoma evansi. Vet J 161:287-300

Weston JF, Heuer C, Williamson NB (2012) Efficacy of a Neospora caninum killed tachyzoites vaccine in preventing abortion and vertical transmission in dairy cattle. Prev Vet Med 103(2):136-144

WHO (World Health, Organization) (2005) A new form of human trypanosomiasis in India. Description of the first human case in the world caused by Trypanosoma evansi. Wkly Epidemiol Rec 80(7):62-63

Wilson M, Jones JL, McAuley JM. (2003) Toxoplasma. In: Manual of clinical microbiology, 8th edn. American Society for Microbiology, Washington, D.C, pp 1970-1980

Wouda W, Moen AR, Visser IR, Van-Knapen F (1997) Bovine fetal neosporosis: a comparison of epizootic and sporadic abortion and different age classes with regard to lesion severity and immunohistochemical identification of organisms in brain, heart, and liver. J Vet Diagn Inv 9:180-185 